\title{
Isolation of Clostridium difficile from human jejunum: identification of a reservoir for disease?
}

\author{
GP TESTORE, F NARDI, S BABUDIERI, M GIULIANO, R DI ROSA, G PANICHI \\ From the Clinica Medica III, Istituto di Anatomia Patologica, Università degli Studi di Roma, "La Sapienza", \\ Rome, Italy
}

SUMmaRY The possibility that the small intestine may represent a reservoir for Clostridium difficile was studied, using segments of human jejunum collected at necropsy. Our results (three of 100 specimens positive for $C$ difficile culture) support the hypothesis that $C$ difficile can be found in human jejunum and that it adheres to the normal mucosa as a resident bacterium. These findings suggest that gastrointestinal disease caused by $C$ difficile has an endogenous origin.

The possibility that the small intestine might represent a reservoir for disease caused by Clostridium difficile was suggested by Taylor et al, when they isolated $C$ difficile from a jejunal aspirate of a patient with chronic colitis. ${ }^{1}$ This hypothesis was confirmed by our experience with a case of pseudomembranous enteritis with spared colon, in which we isolated $C$ difficile from the patient's ileum obtained at necropsy. ${ }^{2}$

To elucidate these findings we carried out a study to verify the rate of isolation of $C$ difficile from human small intestine using segments of jejunum that had been obtained at necropsy.

\section{Material and methods}

\section{COLLECTION OF SPECIMENS}

Over six months one hundred segments of proximal jejunum were collected within 48 hours from 100 patients who had died. The specimens were about $10 \mathrm{~cm}$ long and macroscopically free from lesions. Each segment was placed in a sterile Petri dish and immediately sent to the bacteriology laboratory.

The subjects had died from different diseases, none of them had had diarrhoea or other gastrointestinal symptoms in life. The mean age was 70 years (range $52-86) ; 90 \%$ of the patients had received treatment with antibiotics - that is, $\beta$-lactam antibiotics alone, or in conjunction with aminoglycosides.

\section{PROCESSING OF THE SPECIMENS}

To remove the bacteria that were not firmly attached to mucosa each segment was carefully washed with $10 \mathrm{cc}$ of a sterile saline solution using a vortex mixer for 10 minutes. ${ }^{3}$ This procedure was repeated three

$$
\text { Accepted for publication } 18 \text { March } 1986
$$

times for each sample, changing the container and the washing liquid each time. After this the segment was stretched and the mucosa removed with a sterile lancet; the material obtained was used to inoculate a cycloserine-cefoxitin-fructose selective agar (CCFA) plate. ${ }^{4}$ The plates were screened for colonies characteristic of $C$ difficile; all the cultures were incubated for at least five days before being discarded.

The three washings from each segment were centrifuged for 10 minutes at $5000 \mathrm{rpm}$ and the sediments were used to inoculate a CCFA plate.

\section{Results}

Within 48 hours the cultures from the mucosa were positive for $C$ difficile in three cases. Prolonged incubation of the other samples did not yield any additional positive results. None of the centrifugated washings yielded $C$ difficile. The ages of the culture positive patients were 63,60 , and 74 years; all of them had received treatment with antibiotics.

\section{Discussion}

Although the aetiological role of $C$ difficile in pseudomembranous colitis is now widely accepted, there is still some controversy about the transmission of the disease.

The low percentage of isolation from faeces of healthy adults, ${ }^{5}$ the description of small outbreaks, ${ }^{6-8}$ and the isolation of $C$ difficile from animal reservoirs ${ }^{9}$ favour an exogenous origin of the disease rather than an antibiotic induced overgrowth of undetectable amounts of $C$ difficile that may be already present in the normal intestinal flora.

The isolation of $C$ difficile from a jejunal aspirate of a patient with chronic diarrhoea by Taylor et al and 
the case report of $C$ difficile pseudomembranous enteritis (with large intestinal sparing) that we observed, ${ }^{2}$ made us consider that the jejunum could be a reservoir for disease.

Our results support the hypothesis that $C$ difficile can be found in the jejunum and in subjects without gastrointestinal change; furthermore, the absence of $C$ difficile from washings of anatomical segments indicates that $C$ difficile, when present, adheres to the normal mucosa, which facilitates its integration into the resident flora. These findings suggest an endogenous origin for $C$ difficile mediated gastrointestinal disease.

Interestingly, the rate of isolation $(3 \%)$ found in our work was comparable with that of the isolation of $C$ difficile from the faeces of healthy adults; this raises the possibility that carriers may be colonised throughout the whole of the intestinal tract. To confirm this hypothesis additional studies are required that will evaluate the colonisation of different parts of the intestine by $C$ difficile in the same subject.

\section{References}

${ }^{1}$ Taylor RH, Borriello SP, Taylor AJ. Isolation of Clostridium difficile from the small bowel. Br Med J 1981:283:412.
${ }^{i}$ Testore GP, Pantosti A, Panichi G, et al. Pseudomembranous enteritis associated with Clostridium difficile. Ital J Gastroenterol 1984:16:229-30.

${ }^{3}$ Peach SL, Tabaqchali S. Mucosa-associated flora of human gastrointestinal tract in health and disease. European Journal of Chemotherapy and Antibiotics 1982;2:41-50.

${ }^{4}$ George WL. Sutter VL. Citron D, Finegold SM. Selective and differential medium for isolation of Clostridium difficile. $J$ Clin Microbiol 1978;9:214-9.

${ }^{5}$ George WL, Sutter VL, Finegold SM. Toxicity and antimicrobiol susceptibility of Clostridium difficile, a cause of antimicrobial agent associated colitis. Curr Microbiol 1978;1:55-8.

${ }^{6}$ Mulligan ME, George L. Rolfe RD. Finegold SM. Epidemiological aspects of Clostridium difficile induced diarrhoea and colitis. Am J Clin Nutr 1980;33:2533-8.

${ }^{7}$ Fekety R, Kim K, Brown D, Batts DH, Cudmore M, Silva J. Epidemiololgy of antibiotic-associated colitis; isolation of Clostridium difficile from the hospital environment. $\mathrm{Am} \mathrm{J} \mathrm{Med}$ 1981;70:906-8.

${ }^{8}$ Malamou-Ladas H, Farrell SO, Nash JQ, Tabaqchali S. Isolation of Clostridium difficile from patients and the environment of hospital wards. J Clin Pathol 1983;36:88-92.

${ }^{9}$ Borriello SP. Honour P, Turner T, Barclay F. Household pets as a potential reservoir for Clostridium difficile infection. $J$ Clin Pathol 1983:36:84-7.

Requests for reprints to: Dr Gian Piero Testore, Via Ugo Falena 29, 00137 Roma, Italia. 